Meta

Journal des tradlucteurs

Translators' Journal

\title{
Index des mots et sujets dans le volume 24
}

Volume 24, numéro 4, décembre 1979

URI : https://id.erudit.org/iderudit/003208ar

DOI : https://doi.org/10.7202/003208ar

Aller au sommaire du numéro

Éditeur(s)

Les Presses de l'Université de Montréal

ISSN

0026-0452 (imprimé)

1492-1421 (numérique)

Découvrir la revue

Citer ce document

(1979). Index des mots et sujets dans le volume 24. Meta, 24(4), 504-510.

https://doi.org/10.7202/003208ar d'utilisation que vous pouvez consulter en ligne.

https://apropos.erudit.org/fr/usagers/politique-dutilisation/ 


\section{INDEX DES MOTS ET SUJETS TRAITÉS DANS LE VOLUME 24}

Absorbeur, 3, p. 351.

Abus de confiance, 1, p. 165.

Accents sur les majuscules, 3, p. 415.

Accepteur, 3, p. 365.

Accrédité, 2, p. 276.

Accoutumance, 2, p. 288.

A.C.E.T., 4, p. 496.

Acte de dépôt, 1, p. 213.

Acte de fiducie, 1, p. 211.

Acte de l'état civil, 1, p. 55.

Actes du VIIIe Congrès de la FIT, 3, p. 383.

Acte illocutoire, 1, p. 136.

Action, 1, p. 211.

Action en justice collective, 1, p. 211.

Actionnaire, 1, p. 211.

"Administrer un serment », 1, p. 165 .

Aérogare, 2, p. 303.

Affiche, 4, p. 464

«Agenda 》, 1, p. 163.

Agent cautionné, 1, p. 164

A droite, 4, p. 476.

A gauche, 4, p. 476.

AILA, 4, p. 500.

A la carte, 1, p. 214

Alimentation, 3, p. 393.

Alinéa, 1, p. 58

Allégations fausses, 1, p. 165.

Allogreffe, 4, p. 478.

Allo-immunisation, 4 , p. 478 .

ALPAC (rapport), 3, p. 315.

Aménagement, 1, p. 215.

Aménagement linguistique, 4, p. 494.

Amer (au citron), 3, p. 414.

Amérindiens (noms des peuples), 4, p. 498.

Analyse documentaire, 1, p. 15 .

Analyse du contenu, 1, p. 12.

Analyse du discours, 1, p. 12.

Analyse thématique, 1, p. 12.

Anéjaculation, 4, p. 479.

Année civile, 1, p. 164 .

"Année de calendrier », 1, p. 164.

«Année fiscale », 1, p. 164.

Appel au règlement, 1, p. 163.

Appellation et notion, 4, p. 481.

Appert, 1, p. 32.

Appuyer, 1, p. 163.

Arbitraire du signe (1'), 4, p. 437.

«Argus de l'outil (1') 》, 4, p. 490.

Arséniure de gallium, 3, p. 363.

Association internationale des interprètes de conférence, 3, p. 416.

Association ou société en participation, 1, p. 208.

Associé, 1, p. 209, 210.

Asténozoospermie, 4, p. 479.

Attendu que, 1, p. 161 .

Auteurs, 1, p. 213.

Authentique, 1, p. 165.

Autogreffe, 4, p. 478.
Aviron, 3, p. 415.

« Aviseur légal », 1, p. 160

Avoir du bon temps, 2, p. 241.

Azoospermie, 4, p. 479 .

Bande de conduction, 3, p. 369.

Bande d'énergie, 3, p. 363.

Bande de valence, 3, p. 363 .

Bande interdite, 3, p. 363.

Bande publique (BP), 4, p. 499.

Banque, 2, p. 275.

Banque à charte, 2, p. 274.

Banque commerciale, 2, p. 273.

Banque d'épargne, 2, p. 275.

Banques de terminologie, 2, p. 253,256 ; 4, p. $480,497$.

Banqueroute, 1, p. 161.

Barrière de potentiel, 3, p. 365.

Base, 3, p. 366.

Bâtonnier, 1, p. 215.

Belles-lettres, 1, p. 213.

"Bière, 4, p. 493.

Bière d'épinette, 4, p. 493.

Bière (à la) pression, $3, \mathrm{p} .414$

Bilan, 4, p. 449.

Biotransformation, 2, p. 276.

Biunivocité terminologique, 4, p. 481.

«Boisson aux extraits de gingembre 》, 4, p. 493.

Boisson de régime, 3, p. 414.

Boissons gazeuses, 3, p. $414 ; 4$, p. 492.

Bon père de famille, 1 , p. 38 .

BP, 4, p. 499.

Breveté, 4, p. 498

Brigade, 2, p. 288.

Bruit involontaire, 2, p. 259.

Cache, 1, p. 214.

Canadianismes au Petit Robert, les, 3, p. 395.

Candidature, 4, p. 449.

Canoé, 3, p. 414.

Canot, 3, p. 414.

Capital-actions, 1, p. 208.

Capital déclaré, 1, p. 211.

Capital social, 1, p. 208.

Capter l'énergie, 3, p. 350.

Capteur de brouillard, 4, p. 481.

Capteur solaire, 3 , p. 351 .

Caractère normatif du texte juridique, 1 , p. 39.

Caractériser, 3, p. 411.

Cardiatomie, 4 , p. 479.

Carragénine, 4, p. 479.

Carré au chocolat, 3, p. 415.

Caution, 1, p. 209.

Cautionnement, 1, p. 164.

Cautionnement de soumission, 1, p. 164.

Cellule photoélectrique, 3, p. 351 .

Cellule photovoltaïque, 3 , p. 351 .

Cellule solaire, 3, p. 351 .

Cellulose, 3, p. 414. 
Centre national de la recherche scientifique, 4, p. 491 .

Certificat de prorogation, 1, p. 208.

Certificat de valeur mobilière, 1, p. 211

Certificat d'incorporation, 1, p. 209.

Cession d'un droit, $1, p, 162$.

Chanop sémantique, 1, p. 12.

Chapitre, 1, p. 58.

Charge, 1, p. 160.

Charte, 1, p. 209

Charte de la langue française, 4, p. 494.

Ci-après appelé, 1, p. 161 .

Cidre de frêne, $4, \mathrm{p} .493$.

CILF, 4, p. 491.

Circuit solide, 3 , p. 361.

Citation à comparaître, 1, p. 160 .

\& Cité \$, 3, p. 415.

"Citronade », 4, p. 493.

CNRS, 4, p. 491.

Code, 1, p. 56

Coefficient thermique, 3, p. 364 .

Coinceur à came, 2, p. 276.

Collecteur, 3 , p. 366 .

Collecter l'énergie, 3, p. 350.

Collecteur solaire, 3, p. 351.

Collocation et discours, 3, p. 339.

Commanditaire, 1, p. 211 ; 1, p. 209.

Commission de terminologie de lOOLF, 3, p. 414.

Communication, 1 , p. 10.

Communication linguistique, 4, p. 495.

Commutatrice, 3 , p. 350 .

Compagnie, 1 , p. $164 ; 1$, p. 189

Compagnie aérienne régulière, 2, p. 303.

Compagnie à fonds social, 1, p. $203 ; 1$, p. 210.

Compagnie privée, 1, p. 193 ; 1, p. 209.

Compagnie par actions, 1, p. 203.

Compagnie publique, 1 , p. $193 ; 1$, p. 204 ; 1, p. 210 .

Compagnie statutaire, 1, p. 204.

Composés, 3, p. 364

Compte rendu, 1, p. 163

Concédent, 1, p. 162.

Conclusion, 4, p. 449.

Conditions, 1, p. 161.

Conducteur, 3, p. 363

Conseiller juridique, 1 , p. 160

Constatif, 1, p. 22

Constituer en association, en compagnie en corporation, en société, etc.; incorporer, 1, p. 209

Constitution, 1, p. 71

Contentieux, 1, p. 160.

Contes de Cantorbéry, 3, p. 412

Contrainte du texte juridique, 1, p. 39.

Contraire au règlement, 1, p. 163.

Contre le sens des aiguilles d'une montre, 4, p. 476.

Contrepartie, 1, p. 209

Contrôle, 1, p. 65 .

Convention des actionnaires, 1, p. 211.

Convertir l'énergie, 3, p. 351 .

Copie certifiée conforme, 1, p. 162.

Corédaction, 1, p. 105.

\& Corporation », 1, p. 164, 189, 195, 209.

Corporation de la Couronne, 1, p. 209.

Corporation multiple, 1, p. 194.

Corporation publique, 1, p. 210.
Corporation simple, 1,p: 194.

Corporatisme 1, p. 195

Corporatiste, 1, p. 195.

Corps constitué, 1, p. 209.

Corps simple, 3, p. 362, 364.

Couche, 3 , p. 362 .

Couche d'énergie, 3, p. 362.

Couche de valence, 3 , p. 362.

Cour, 1, p. 32.

Courtier attitré, 1, p. 210.

Coutume (a), 1, p. 72

C.Q.F.D., 1, p. 32 .

Créance, 1, p. 210.

«Crème », 4, p. 493.

Créneau-vacances, 2, p. 303.

Croissance dentritique, 3 , p. 350 .

Curé, 1, p. 213, 215 .

Dame, 1, p. 32

Dans le sens d'horloge, 4, p. 476.

D'autre part, 1, p. 161 .

De bonne foi, 1, p. 165.

De droite à gauche, 4, p. 476.

De gauche à droite, 4, p. 476.

Débile psychopathe, 1, p. 154.

Déclaration sous serment, 1, p. 165.

«Décrétales », 1, p. 147.

Décrire, 3, p. 411 .

Définition, problème de la, 1, p. 12

Défricher, 3, p. 411.

Dégager, 3, p. 411.

\&Déjudiciarisation \$, 1, p. 142

Délibéré, 1, p. 213.

Dénomination sociale, 1, p. 168, 209.

«Département légal », 1, p. 260.

Descriptivisme linguistique, 4, p. 481.

Détente, 4, p. 499.

Détenteur, 3, p. 370

Détenteur (liste sélective des cooccurrents), 3, p. 373.

Dette, 1, p. 210.

Dévésiculeur, 4, p. 481

Déviance, 1, p. 142.

Dévissé, 4, p. 477.

Dextrosum, 4, p. 476.

Diagramme énergétique, 3, p. 363.

《Dialecte social \$, 1, p. 11.

Dictionnaire automatique, 2, p. 258.

Diffamation, 1, p. 160.

Diode à cristal, 3, p. 366.

Diode semiconductrice, 3, p. 366.

Diplomatie dans la révision, 4, p. 497.

Directeur du Bureau des véhicules automobiles, 1, p. 216.

Discours, 1, p. 34 .

Discours géographique, 1, p. 29.

Discours grammatical, 1, p. 28.

Discours juridico-politique, 1, p. 133.

Discours juridique, 1, p. 26.

Discours et sémantique, 3 , p. 336.

Dissolution, 1, p. 209.

Distributeur d'essence, 4, p. 499.

Doctrine, $1, \mathrm{p} .73$.

Donneur, 3, p. 365.

Dopage, 3, p. 365.

Dotation, 4, p. 499.

Drogue, 4, p. 478.

Droit, 1, p. 23, 37, 70 .

Droit anglais, 1, p. 74 .

Droit britannique, 1 , p. 75. 
Droit civil, 1, p. 72, 207.

Droit commun, 1 , p. 70 .

Droit de gage, 1, p. 99.

Droit de rétention, 1, p. 160.

Droit d'hypothèque, 1, p. 99.

Droit français, 1, p. $46,69,74$.

Droit international, 1, p. 125.

Droit pénal, 1, p. 24.

Droit privé, 1, p. 70.

Droit public, 1, p. 70 .

D'une part, 1, p. 161.

Eau gazéifiée, 3, p. 414.

Echecs traductionnels, 4, p. 441

Edifice public, 1, p. 80.

Effet indésirable, 4, p. 479.

Effet photoélectrique, 3, p. 364.

Effet \& serendip $», 1$, p. 41 .

Effet transistor, 3, p. 367 .

Electroluminescent, 3, p. 364.

Electrons de valence, 3, p. 362 .

Electrons-volts, 3, p. 363.

Elément, 3, p. 362 .

Eliminateur de brouillard, 4, p. 481.

Eliminateur de gouttelettes, 4, p. 481.

Emetteur, 3, p. 366 .

Employé, 1, p. 119.

En bloc, 1, p. 214.

En brevet, 1, p. 213

En droit, 1, p. 164.

En entier, 1, p. 214

En fait, 1, p. 164.

En foi de quoi, 1, p. 161

En minute, 1, p. 213.

Enr., 1, p. 210

Enregistré, 1, p. 210.

Enregistrement, 1, p. 210.

Enseigne, 4, p. 464.

En sens d'horloge, 4, p. 476.

Entraîner, 4, p. 449.

Entreprise, langue de 1', 3, p. 411.

Eprouver, 4, p. 449

Escroquerie, 1, p. 165.

Essarter, 3, p. 411.

Estimateur, 1, p. 208 .

Etat, 1, p. 31 .

Etat de manque, 4, p. 478 .

Et Cie, 1, p. 210.

Ethique, les collocables du mot, 3, p. 345 .

Etiquetage industriel, 4, p. 498.

Etiquette, 4, p. 464

Euroducatum, 3, p. 322.

$\mathrm{eV}, 3$, p. 363 .

Exercice, 1, p. 164.

Existence d'un corps constitué, d'une société, 1, p. 209.

Explication de texte (méthode de 1'), 4, p. 491.

Exposer, 3, p. 411.

Extrinsèque, 3, p. 364 .

(fabrication/production) sous licence, 1, p. 162.

Fabrique, 1, p. 213.

Fabriqué à/ou/en, 4 , p. 498.

Facteurs extra-linguistiques, 4, p. 494.

Faillite, 1, p. 161.

Faire attention, 2, p. 241.

Faire prêter serment, 1, p. 165.

Fait de discours, 1, p. 33 .
Fédération internationale des traducteurs, $4, p .500$.

Féminin dans les appellations d'emploi, 4, p. 498.

Féminin des noms de peuples amérindiens, 4, p. 498

FID, 4, p. 500 .

Fiduciaire, 1, p. 211

Fiducie, 1, p. 97

FIT, 4, p. 500.

Fleurdelisé, 1, p. 214

Flexible de distribution, 4, p. 499.

Fondateur, 1, p. 210.

Fondateur (d'une société), 1, p. 209.

Fondé de pouvoir, 1, p. 210.

Fonds capital, 1, p. 203.

Fonds commun, 1, p. 203.

Fonds social, 1, p. 203.

Forfait, 2, p. 303.

Forfait avion-auto, 2, p. 303

Forfait avion-bateau, 2, p. 303.

Forfait golf, 2, p. 303.

Forfait hôtelier, 2, p. 303.

Forfaitiste, 2, p. 303 .

Formation universitaire, 4, p. 423.

Francisation du Québec, 4, p. 494.

«Frênette », 4, p. 493.

Garagiste, 1, p. 215.

Garde, 1, p. 209.

"Gazette officielle \#, 4, p. 492.

Gouvernement, 1, p. 31 .

Greffe allogénique, 4, p. 478.

Greffe usogénique, 4, p. 478 .

\& Gouvernement de fait », 1, p. 133.

Hélice à gauche, 4, p. 476.

Héliopile, 3, p. 351 .

Hétérogreffe, 4 , p. 478.

Hétérophile, 4, p. 478.

Histocompatibilité, 4 , p. 479

Homogreffe, 4, p. 479.

« Hors d'ordre $\$, 1$, p. 163

《Huile à/de chauffage », 4, p. 499.

« Huile à fournaise $», 4$, p. 499.

Hypospermie, 4, p. 479.

Hypothèque, 1, p. 112 .

Idéologie $\left(1^{\prime}\right), 1$, p. 132.

ILF, 4, p. 491 .

Immatriculation, 1, p. 210.

Imprimé à/ou/en, 4, p. 498.

Impureté, 3, p. 365.

"Incorporation », 1, p. 192.

Incorporée - ou - Inc., 1, p. 209.

Index, 1, p. 11.

Industries alimentaires, 3, p. 393.

Infoterm, 4, p. 497.

Initié, 1, p. 210.

Inscription, 1, p. 210.

Institut de la langue française, 4, p. 491.

Intégration à grande échelle, 3, p. 361 .

Interaction sur le plan horizontal, $4, p$. 437.

Interaction sur le plan vertical, 4, p. 437.

Interprétation, 3, p. 389.

Interprétation judiciaire, 1, p. 115.

Interprétation juridique, l'art de 1, 1, p. 19.

Interrogation indirecte, 1, p. 31

Intrinsèque, 3, p. 364 .

Irrecevable, 1, p. 163 .

Irrégulier, 1, p. 163. 
Iso-imumunisation, 4, p. 479.

Isogreffe, 4 , p. 479 .

Isolant, 3, p. 363.

«Item sur l'agenda », 1, p. 163.

Jonction PN, 3, p. 365 .

Journée de la révision, 4 , p. 496.

Journée de traduction, 4, p. 423.

Jugement, 1, p. 24.

Jurisprudence, 1 , p. 73.

JURIVOC (le projet), 1, p. 109.

Lacune, 3, p. 365.

Langage, 1, p. 19, 36 .

Langage diplomatique, 1, p. 124.

Langage du droit, 1, p. 42.

Langage juridique, 1, p. 19.

Langage juridique, fonctions, 1, p. 20.

Langage juridique, structure, 1, p. 21.

Langue, 1, p. 36.

«Langue bourgeoise », 1, p. 13.

* Langue campagnarde $», 1$, p. 13.

Langue canonique, 1 , p. 147.

Langues de spécialité, 4, p. 488.

Langue du droit, 1, p. 13 .

Langue et parole, 4, p. 437.

"Langue noble ", 1, p. 13.

"Langue prolétarienne », 1, p. 13.

La solution apportée au problème, 4, p. 449.

Latin (le), 1, p. 148.

Le, 1, p. 61

Ledit, 1, p. 32.

Les clonnées du problème, 4, p. 449.

Législation dite déléguée, 1, p. 71 .

Lettres patentes, 1, p. 210 .

« Let's talk D.P., Lexique d'informatique, français/anglais, américain $\gg, 4, p .488$.

Levier, 4, p. 499.

Lexicographie juridique, 1, p. 49.

Lexicologie et terminologie, 4, p. 480.

«Lexique des boissons gazeuses ", 4, p. 492 .

Lexis., 3, p. 322.

Lexie et discours, 3, p. 338.

《Libelle 》, 1, p. 160.

《Lien 》, 1, p. 160

Ligne aérienne, 2, p. 303.

Limitée - ou - Ltée, 1, p. 209.

Limonade, 3 , p. 414 ; 4, p. 494.

Linguistique diachronique, 1, p. 14.

Linguistique et sciences juridiques, 1, p. 9.

Liquidation, 1, p. 211.

Liquider, 1, p. 211.

«Libre de l'outil », 4, p. 490.

Locutions latines, 1, p. 32.

Loi (la), 1, p. 71 .

Loi 101,4, p. 495

Lsp, 4, p. 488 .

Mainlevée, 1, p. 213.

Mandataire, 1, p. 210.

Manuel, 4, p. 459.

Manuel de littérature, 4, p. 459.

Manuel de philosophie, 4, p. 459

Manuel de physique, 4, p. 459.

Manuel d'instructions, 4, p. 459.

《 Manuel pratique de terminologie », 4, p. 486.

Marche/Arrêt, 4, p. 499.

Marquer, 3, p. 411.

Matériaux semiconducteurs, 3, p. 364.
Mazout, 4, p. 499.

"Mépris de cour », 1, p. 160.

Message, 4, p. 437.

Métalangage, 1, p. 28.

Métaphore, 4, p. 436.

Métonymie, 4, p. 436

Mettre en manchette, 3, p. 411.

Micro-électronique, 3, p. 388.

Micro-informatique, 3 , p. 388 .

Microrragie, 4, p. 479.

Ministre du tourisme, de la chasse et de la pêche, 1, p. 217.

《 minutes $\$, 1$, p. 163

Mis en opposition, 3, p. 366.

Modérateur, 1, p. 156 .

Montage à base commune, 3, p. 367

Montage à collecteur commun, 3, p. 367.

Montage à émetteur commun, 3, p. 367.

Mot de liaison, 1, p. 32.

Mot-terme, 3, p. 336 .

Motelier, 2, p. 303.

Mots images, 4, p. 435.

Mots signes, 4, p. 435 .

Mots images et mots signes, 4, p. 435 .

Motion, 1, p. 163

Mutateur, 3, p. 350 .

Nécrozoospermie, 4, p. 479.

Nectar, 3, p. 414.

Néonatologie, 4, p. 480.

Niveau, 3 , p. 362 .

Niveaux de langue, 4, p. 495.

Niveau d'énergie, 3, p. 362.

Nom commercial, 1, p. $169,208$.

«Nom corporatif $», 1$, p. 174 .

Nombre atomique, 3, p. 362.

Nombre quantique, 3, p. 362.

Nombre de valence, 3, p. 362.

Nomenclature, 1 , p. 26

Nonobstant, 1, p. 32.

Normalisation terminologique, 2, p. 258.

Normalisation et terminologie, 3, p. 353.

Normalisation, dossier de, 3, p. 354.

Normalisation, parties du dossier de, 3, p. 354.

Normalisation terminologique, 4, p. 481.

Norme objective (ling.), 4, p. 495.

Norme personnelle (ling.), 4, p. 495.

Norme sociale (ling.), 4, p. 495.

Notamment, 1, p. 32 .

Notice, 4, p. 459 .

Notice de fonctionnement et d'entretien, 4 , p. 459.

Notice de montage et d'installation, 4, p. 459.

Notice d'entretien, 4, p. 459.

Notice technique, 4, p. 459.

Notion à appellation, 4, p. 481 .

Numéro atomique, 3, p. 362.

Obligation, 1, p. 210

Obligation juridique, 1 , p. 38

Office de la langue française, 4, p. 492.

Offre d'achat visant à la mainmise, $1, \mathbf{p}$. 211.

OLF, 4, p. 492.

Oligozoospermie, 4, p. 479.

Onduleur, 3, p. 350.

Opposition distinctive, 1, p. 10.

Ordre du jour, 1, p. 163.

Orangeade, 3 , p. 414 
Outils anciens, 4, p. 490.

Outrage au tribunal, 1, p. 160.

Ouvrages de référence, 4, p. 491.

Ouvroir, 1, p. 215.

Pagaie, 3, p. 415.

Palan pneumatique à piston, 3 , p. 343 .

Pancarte, 4, p. 464.

Panneau, 4, p. 464.

Paragraphe, 1, p. 58.

Paramètre : constante ou discours, $3, \mathrm{p}$. 343.

Paravent de la société, 1, p. 209.

Pari mutuel, 1, p. 214.

Parrain, 1, p. 163.

"Partie de première part », 1, p. 161.

«Partie de seconde part $», 1$, p. 161.

Passif (traduction du), 2, p. 241.

Passif, 1, p. 210.

Patrimoine d'une société, 1, p. 208.

Patronage, 1, p. 215.

Performatif, 1, p. 11, 22.

Périnatologie, 4, p. 480.

Personne morale, 1, p. 55, 178, 208, 209, 210.

Personne physique, 1, p. 210.

Pertinence des sons, 1, p. 9.

Pertinent, 1 , p. 9.

Pertinents, 1, p. 165.

Pesticide, 3, p. 415. Petit Larousse illustrế 1980 (ajouts), 4, p.

Petit Robert 1978, 3, p. 395.

Peuples amérindiens (graphie des noms de), 4, p. 498.

Pharmacocinétique, 2, p. 276.

Pharmacodépendance, 4, 478 ; 2, p. 288.

Pharmacodynamie, 2, p. 276.

Philosophie du droit, 1, p. 19.

Phonologie, 1, p. 9.

Photoélectrique, 3, p. 364.

photo-émetteur, 3, p. 364.

Pile photoélectrique, 3 , p. 351

Pile photovoltaiqque, $3, \mathrm{p} .351$.

Pile solaire, 3, p. 351 .

Pistolet, 4, p. 499.

Plaire à, 2, p. 241

Planification linguistique, 4, p. 494.

Plaque, 4, p. 464.

Pluriel des noms de peuples amérindiens, 4, p. 498.

Point à/de l'ordre du jour, 1, p. 163.

«Point d'ordre 》, 1, p. 163.

Polarisé en direct, 3, p. 366.

Polarisé en inverse, 3 , p. 366.

Pollicitant, 1, p. 210.

Pollicitation, 1, p. 210.

Polyzoospermie, 4, p. 479.

Porteur, 3, p. 370.

Porteur (liste sélective de cooccurrents), 3, p. 373.

Poser un problème, 4, p. 449.

Post-édition, besoins et prix de, en traduction automatique, 3, p. 319.

Préconiser, 4, p. 449

Prendre soin, 2, p. 241.

Présent, 1, p. 65.

Présomption, 1, p. 209.

Présomption de livraison, 1, p. 209.

Présomption de propriété, 1, p. 209.
Présuppositions du discours, 1, p. 130.

Pression, 4, p. 449.

Principe d'exclusion de Pauli, 3, p. 363.

Privilège, 1, p. 210.

Problèmes de la traduction juridique au Québec, 1, p. 35.

Procès-verbal, 1, p. 213, 163.

Procuration, 1, p. 210.

Promoteur, 2, p. 303.

Proposer, 1, p. 163.

Proposeur, 1, p. 163.

Proposition, 1, p. 163.

Propriété, 1, p. 14, 98.

Propriété effective, 1, p. 208.

Prorogation, 1, p. 209.

Provision, 1, p. 160.

Psychiatrie, 2, p. 289.

Publiciste, 2, p. 303.

Publicitaire, 2, p. 303.

Puce, 3, p. 367.

Pureté, 3 , p. 364.

Qualité de la langue française, 4, p. 495.

Qualité externe de la langue, 4, p. 495.

Qualité intrinsèque de la langue, 4, p. 495.

Rachat (des actions), 1, p. 210.

Racinette, 3, p. $414 ;$ 4, p. 493.

Radiotélévision, 4, p. 499.

Raison (1a), 1, p. 74.

Raison commerciale, 1, p. 169.

Raison de commerce, 1, p. 169.

Raison sociale, 1, p. 166, 209.

Randonneur, 2, p. 303.

Réacteurs nucléaires (résumé terminologique), 4, p. 463.

Recherche documentaire terminologique, 3 , p. 354.

Recherche métaphysique, 1, p. 19.

Recherche thématique: choix des documents de base, 3, p. 354 .

Recours collectif, 1, p. 211 .

Recouvrement, 1, p. 210.

Recueillir l'énergie, 3, p. 350.

Rédaction et terminologie, 3, p. 352.

Rédaction et vocabulaire de soutien, $1, \mathbf{p}$ 27.

Rédaction juridique, difficultés de la, $1, \mathbf{p}$. 47.

Redresseur, 3, p. 350.

Redresseur à semiconducteur, 3, p. 366.

Réflexion sur le discours juridique, 1, p. 26.

Régate (lexique anglais-français), 4, p. 465 .

Régistre de la corporation, de la société, 1 , p. 209.

Règle du précédent (la), 1, p. 76.

Règlement, 1, p. 71, 208 .

Règlement intérieur, 1, p. 164.

Regroupement d'entreprises, 1, p. 208.

Relativité du sens des mots, 1, p. 131 .

Renonciation, 1, p. 211, 162 .

Repeindre, 3, p. 411.

\& Répertoire des dictionnaires scientifiques et techniques 1950-1975 », 4, p. 491 .

Représentant, 1, p. 209.

Représenter, 3, p. 411

Résistance de transfert, 3, p. 361.

Résistance spécifique, 3, p. 363.

Résistivité, 3, p. 363

«Résolution », 1, p. 163.

Résoudre un problème, 4 , p. 449. 
Responsabilité civile, 1, p. 209.

Responsabilité limitée, 1, p. 210

Restaurant-minute, 2, p. 303.

Réviseurs et traducteurs (rapports entre), 4, p. 496.

Révision, 3, p. 385.

Révision collective, 4, p. 496

Révision externe, 4, p. 428.

Révision réciproque, 4 , p. 496.

Rôle sémantique du contexte français, 4, p. 438.

《Routebire », 4, p. 493.

S.A., 1, p. 210

«Sapinette 》, 4, p. 493.

Saut, 2, p. 303.

Sceau de la compagnie, 1, p. 209.

Schéma de principe, 3, p. 367.

Scrip, 1, p. 211.

Se consacrer, 4 , p. 449

«Se faire intenter une action en libelle », 1, p. 160 .

Se faire intenter un procès en diffamation, $1, p, 160$.

Se heurter à un problème, 4 , p. 449 .

Second parrain, 1, p. 163.

«Seconder $\gg, 1$, p. 163.

«Secondeur $\$, 1$, p. 163

Secrétaire juridique, 1, p. 160.

«Secrétaire légal(e) », 1, p. 160.

Section, 1, p. 58.

Section de terminologie, 3, p. 415.

Selon la première éventualité, 1, p. 161.

Selon le montant le plus bas, 1, p. 161.

Selon le montant le plus élevé, 1, p. 161.

Sème, 1, p. 13 .

Semiconducteur, 3, p. 363.

Semiconducteurs (microlexique), 3, p. 368

Semiconducteur du type N, 3, p. 365 .

Semiconducteurs extrinsèques, 3, p. 364

Semiconducteurs intrinsèques, 3, p. 364.

Sémiotaxie, 3, p. 341.

Senestrorsum, 4, p. 476

Sens antihoraire, 4, p. 476.

Sens des aiguilles d'une montre, 4, p. 476.

(Sens) dextrogyre, 4, p. 477.

Sens direct, 4 , p. 476 .

Sens droite, 4, p. 477.

Sens du mouvement des aiguilles d'une montre, 4, p. 476

Sens giratoire gauche, 4, p. 477.

Sens horaire, 4, p. 476.

Sens inverse de celui des aiguilles d'une montre, 4 , p. 476

Sens inverse d'horloge, 4 , p. 476

Sens inverse du mouvement des aiguilles d'une montre, 4, p. 476.

(Sens) lévogyre, 4 , p. 477.

Sens «négatif $», 4$, p. 476,477 .

Sens « normal », 4, p. 476.

Sens opposé aux aiguilles d'une montre, 4, p. 476.

Sens opposé des aiguilles d'une montre, 4 , p. 476 .

Sens « positif », 4, p. 476 .

Sens rétrograde, 4, p. 476.

Sens trigonométrique, 4 , p. 477

Sens global, 4, p. 437.

Sens inverse, 4, p. 477.

Séparateur de gonttes, 4, p. 481
Séquestre, 1, p. 210.

Service(s) juridique(s), 1, p. 160 .

Servitude, 1, p. 100, 160 .

Sevrage, 4, p. 478.

Siège social, 1, p. 209, 210.

Sieur, 1, p. 32

Signature sociale, 1, p. 209.

Signe, 1, p. 16.

Signifiant et signifié, 4, p. 434.

Silence involontaire, 2, p. 259.

Sinistrorsum, 4, p. 476

Situation, 1, p. 164

Société, 1, p. 164, 188, 211.

Société anonyme, 1, p. 168, 183, 204, 208, 210.

Société à responsabilité limitée, 1, p. 184.

Société à responsabilité limitée - ou S.A.R.L., 1, p. 209.

Société à responsabilité limitée - ou - anonyme, 1 , p. 210.

Société ayant fait appel au public, 1, p. 209.

Société cautionnée, 1, p. 164.

Société « collective $\$, 1$, p. 183.

Société commerciale, 1, p. 182

Société de capitaux, 1, p. 183, 185.

Société de cautionnement, 1, p. 164

Société de garantie, 1 , p. 164 .

Société de la Couronne, 1, p. 209.

Société de personnes, 1, p. 185 .

Société de personnes à responsabilité limitée, 1 , p. 210

Société des traducteurs du Québec, 3, p. 414.

Société d'Etat, 1, p. 209.

Société d'une personne, 1, p. 189.

Société en commandite, 1 , p. 182 .

Société en commandite (simple), 1, p. 210

Société en nom collectif, 1, p. 171, 177, $209,210$.

Société fermée, 1, p. 187, 209.

«Société générale 》, 1, p. 183.

Société ouverte, 1, p. 187, 210

Société par actions, 1, p. 202, 210.

Société par actions non incorporée, 1, p. 203.

Société par intérêts, 1, p. 183.

Société universonnelle, 1, p. 188.

Soda, 3, p. $414 ; 4$, p. 493.

"Soda à la vanille », 4, p. 493.

"Soda au sassafras $», 4$, p. 493.

Soda de régime, 3 , p. 414 .

Soda (au) gingembre, 3, p. 414 ; 4, p. 493.

Soda (à l')épinette, 3, p. 414.

Soda gingembre piquant, 3, p. 414

Soda hypocalorique, 3, p. 414.

Soda mousse, 3, p. 414 ; 4, p. 493.

Soda (à $1^{\prime}$ )orange, 3, p. 414.

Soda piquant au gingembre, 3, p. 414.

Soda racinette, 3 , p. 414

Soda tonique, 3 , p. 414.

S.P.A.R.L., 1, p. 210

Soumission cautionnée, 1, p. 164.

Sources du droit, 1, p. 72

Sous réserve des droits, 1, p. 165.

Sons-section, 1, p. 58.

Sous toutes réserves, 1, p. 165.

Spécial, 1, p. 165.

Stages, 4, p. 423 
Stages externes, 4, p. 430.

Stages internes, 4, p. 428 .

Statut(s), 1, p. 164, 208, 210.

S.T.Q., 4, p. 496.

Stocker l'énergie, 3, p. 350.

Structure performative, 1, p. 24.

Stupéfiant, 4 , p. 478.

Stylistique, 1, p. 13.

Stylistique des effets, 1, p. 14.

Stylistique génétique, 1, p. 14.

«Sub poena $», 1$, p. 160.

Substances photo-émettrices, 3, p. 364.

Substances photo-émissives, 3, p. 364.

Surintendant des assurances, 1, p. 216.

Suivi (d'une affaire), 1, p. 28.

Suivre, 1, p. 28.

Synapsie, 3, p. 337.

Synecdoque, 4, p. 436.

Syntagme/collocable, 3, p. 339.

Syntagme implicite , 4 , p. 436.

Syntagme et synapsie, 3, p. 337

Syntagmation et discours, 3, p. 340 .

Syntagmation, les opérations sémantiques de la, 3, p. 340.

Symposium de la Fondation Nobel, 39e, 3, p. 376.

Système de traduction automatique, 3 , p. 316.

Système informatisé d'aide aux traducteurs, 3, p. 322.

Table d'hôte, 1, p. 214.

Tension directe, 3, p. 365 .

Tension inverse, 3, p. 366 .

Terme, 2, p. 254 .

《Termes et conditions 》, 1, p. 161.

Terme-mot, 3, p. 336.

Terme technique, 1, p. 26.

Terminologie, 2, p. 254

Terminologie : corps de vocabulaire, $3, \mathrm{p}$. 352.

«Terminologie» (diverses acceptions), 4, p. 486.

Terminologie de l'association ACP-CEE, 3, p. 379.

Terminologie: Dossier de normalisation, 3 , p. 354.

Terminologie du Common Law, 1, p. 181.

Terminologie: Etude synonymique, 3, p. 352.

Terminologie et lexicologie, 4, p. 480.

Terminologie juridique, 1, p. 111 .

Terminologie juridique des Communautés européennes, 3 , p. 378.

Terminologie, les champs d'application de la, 3, p. 349.

Termium, 3, p. 322 .

Terminus urbain, 2, p. 303.

Tetratozoospermie, 4, p. 479

Tétravalent, 3 , p. 364 .

Théorie de Coode, 1, p. 86.

Théorie des actes de discours, 1, p. 22.
Tirage en filières, 3, p. 350 .

Titre, 1, p. 58.

Titulaire, 3, p. 370.

Titulaire (liste sélective des cooccurrents), 3, p. 373 .

Titulaire d'une licence, 1, p. 162.

Tolérance, 4, p. 478

Tourismologie, 2, p. 303.

Tourner, 3, p. 411 .

Tournure négative, 1, p. 88.

Tous droits réservés, 4, p. 498.

Tout(e), 1, p. 61 .

Toxicomanie, 4 , p. $478 ; 2$, p. 288.

«Tradioscopie $\$, 4$, p. 426

Traducteur des affaires extérieures, $1, \mathbf{p}$ 127.

Traduction automatique, fautes de, $3, p$. 320.

Traduction automatique, les problèmes de la, 3, p. 315 .

Traduction automatique: perspectives d'avenir, 3 , p. 315.

Traduction automatique, rendement de, 3 p. 325.

Traduction, problème de la, 1, p. 35 .

Traduction canonique, 1 , p. 146 .

Traduction des lois fédérales, 1, p. 104.

Traduction directe, 2 , p. 245 .

Traduction et terminologie, 3, p. 349 .

Traductions impossibles, 4, p. 441.

Traduction indirecte, 2, p. 243.

Traduction juridique et son enseignement, 1, p. 35.

Traduction littérale (problème de la), 1, p. 99.

Traduction technique, 1, p. 95.

Travail, les collocables de, 3 , p. 345.

Trou, 3, p. 365.

Trust, 1, p. 97, 181.

Tutoiement, 1, p. 29

Type P, 3, p. 365 .

Un, 1, p. 62

Unification du droit, 1, p. 20.

Unité du droit, 1, p. 20.

Utilisateurs de terminologie, 4, p. 486.

Ventes à découvert, 1, p. 211 .

Vérificateur, 1, p. 208.

Véritable propriétaire, 1, p. 208.

Versification, 1, p. 213.

Veuve, 1, p. 32 .

Ville, 3, p. 415 .

Vissé, 4, p. 477

Vocabulaire de soutien, 1, p. 26.

Vocabulaire d'encadrement, 1, p. 28.

Vocabulaire général d'orientation scientifique (V.G.O.S.), 1, p. 28.

Vocabulaire paratechnique, 1, p. 27.

《Voir clair», 1, p. 154.

Vote cumulatif, 1, p. 209.

Voyagiste, 2, p. 303.

Xénobiotique, 4, p. 480

Xénogreffe, 4, p. 479. 